\title{
A Parimutuel Market Microstructure for Contingent Claims
}

\section{Jeffrey Lange}

Longitude Inc.

email: jlange@longitude.com

\section{Nicholas Economides}

Stern School of Business, New York University and NET Institute email:neconomi@stern.nyu.edu

\begin{abstract}
Parimutuel principles are widely used as an alternative to fixed odds gambling in which a bookmaker acts as a dealer by quoting fixed rates of return on specified wagers. A parimutuel game is conducted as a call auction in which odds are allowed to fluctuate during the betting period until the betting period is closed or the auction 'called'. The prices or odds of wagers are set based upon the relative amounts wagered on each risky outcome. In financial microstructure terms, trading under parimutuel principles is characterised by (1) call auction, non-continuous trading; (2) riskless funding of claim payouts using the amounts paid for all of the claims during the auction; (3) special equilibrium pricing conditions requiring the relative prices of contingent claims equal the relative aggregate amounts wagered on such claims; (4) endogenous determination of unique state prices; and (5) higher efficiency. Recently, a number of large investment banks have adopted a parimutuel mechanism for offering contingent claims on various economic indices, such as the US Nonfarm payroll report and Eurozone Harmonised inflation.

Our paper shows how the market microstructure incorporating parimutuel principles for contingent claims which allows for notional transactions, limit orders, and bundling of claims across states is constructed. We prove the existence of a unique price equilibrium for such a market and suggest an algorithm for computing the equilibrium.

We also suggest that for a broad class of contingent claims, that the parimutuel microstructure recently deployed offers many advantages over the dominant dealer and exchange continuous time mechanisms.
\end{abstract}

We would like to thank Ken Baron of Longitude, Darrell Duffie of Stanford University, and Michael Overton of the Courant Institute at NYU for providing critical insight and support. This article is based on Lange and Economides (2001). Proofs that are omitted in this published version can be found in Lange and Economides (2003) at http://www.stern.nyu.edu/networks/ Parimutuel.pdf 


\section{Introduction}

Parimutuel principles were invented in late nineteenth century France by Pierre Oller as an alternative to the bookmaker syndicates that dominated French gaming at the time. The parimutuel mechanism supplanted bookmaker horse racing in the USA beginning in the 1920s and 1930s facilitated in large part by the invention of the automatic odds calculator (or 'totalisator') by Harry Strauss. ${ }^{1}$

Recently, a number of large investment banks have adopted a parimutuel mechanism for offering contingent claims on various economic indices, such as the US Nonfarm payroll report, Eurozone Harmonised inflation, and Fannie Mae mortgage pool prepayment speeds. The parimutuel mechanism employed is a call auction lasting about one hour for claims on the underlying index which include a variety of standard and exotic derivatives, including vanilla call and put options, forwards, digital options, range binary options, and linked buy/sell options such as risk reversals. A unique feature of the microstructure is that all of the claims offered are priced in equilibrium based upon an implementation of parimutuel mechanism principles. Our aim is to formalise these principles and point out some of the inherent advantages of the mechanism as applied to the recent auctions.

As a market microstructure, the parimutuel mechanism has four distinguishing features: (1) the parimutuel mechanism is a call auction market rather than a continuous auction; (2) relative prices of contingent claims are equal to the relative aggregate cost of such claims; (3) the total amount paid for the contingent claims is exactly sufficient to pay for the contingent claims having a positive return, that is, the mechanism is self-funding and risk-neutral in the sense that the total premium paid for contingent claims is equal to the state contingent payouts for all contingent claims expiring 'in-the-money; ${ }^{2}$ (4) a unique set of endogenously determined prices is discovered; and (5) higher efficiency than other trading mechanisms.

Our approach is to formally provide a foundation for the parimutuel mechanism and then describe in detail the mechanism recently employed in the capital markets. Our first step then is showing a foundational connection between parimutuel principles and the theory of market games. In Section 2, we show that a parimutuel contingent claims market is a natural extension of a Shapley-Shubik market game for contingent claims. ${ }^{3}$ Thus, we connect the parimutuel mechanism to the welldeveloped market games literature and show that a parimutuel mechanism is a viable mechanism for a contingent claims market with endogenous price formation. In Section 3, we discuss in detail the parimutuel market microstructure recently employed to offer contingent claims on the Eurozone inflation index, US economic statistics such as the nonfarm payroll releases, Fannie Mae mortgage pool prepayment speeds and other indices. We show that the parimutuel microstructure with notional claims, limit orders, and 'claim bundling' across states has a unique price equilibrium. We also present a theorem which shows that all parimutuel mechanisms can be expressed as a solution to a general eigenvalue problem. Section 4 discusses the

\footnotetext{
${ }^{1}$ Considerable empirical work has been done on the efficiency and information characteristics of parimutuel wagering. See Haush et al. (1994).

${ }^{2}$ In this paper, we ignore transaction costs which can be quite significant in parimutuel gambling contexts.

${ }^{3}$ See Shapley and Shubik (1977).

(C) Blackwell Publishing Ltd, 2005
} 
efficiency and no-arbitrage characteristics of the parimutuel microstructure as applied to the capital markets. In particular, we show that the liquidity aggregation features of the parimutuel microstructure, both across time in a call auction and across disparate types of contingent claims, can reduce the amount of noise around the fair price of such claims. Section 5 concludes.

\section{Parimutuel Microstructure and Market Games}

Parimutuel principles are widely used as an alternative to fixed odds gambling in which a bookmaker acts as a dealer by quoting fixed rates of return on specified wagers. A parimutuel game is conducted as a call auction in which odds are allowed to fluctuate during the betting period until the betting period is closed or the auction 'called'. The prices or odds of wagers are set based upon the relative amounts wagered on each risky outcome. In microstructure terms, wagering under parimutuel principles is characterised by (1) call auction, non-continuous trading; (2) riskless funding of claim payouts using the amounts paid for all of the claims during the auction; (3) special equilibrium pricing conditions requiring the relative prices of contingent claims equal the relative aggregate amounts wagered on such claims; and (4) endogenous determination of unique state prices.

When applied to the theory of contingent claims markets, the self-funding and relative pricing features of a parimutuel system result from the guaranteed existence of a positive state price vector, $\mathbf{p}$, which excludes arbitrage over the state space. ${ }^{4}$ The vector $\mathbf{p}$ contains the prices for each elemental state outcome.

We will show that the existence of the positive state price vector combined with enforcing the equality of the aggregate payouts for each state are sufficient to guarantee that contingent claims are both self-funding and that the relative prices of claims are equal to the relative amounts paid for such claims. Assuming no transaction costs, and for purposes of this discussion, zero interest rates, the absence of arbitrage requires the following normalisation condition on the state prices:

$$
\mathbf{p}^{T} \mathbf{e}=1, \mathbf{p}>0
$$

where $\mathbf{p}$ is a strictly positive $S$-dimensional vector of state prices (probabilities), e is an $S$-dimensional unit vector, and superscript $T$ is the familiar transpose operator. Multiplying by a vector $\mathbf{y}$, an $S$-dimensional vector containing the aggregate state payouts for each state, yields the riskless condition that all payouts are identical across the states:

$$
\left(\mathbf{y}^{T} \mathbf{p}\right) \mathbf{e}=\mathbf{y}
$$

Since the left-hand side of (2) is a vector containing the aggregate premium investment, (2) states that the state contingent payout of each state is equal to the aggregate

\footnotetext{
${ }^{4}$ We employ the term state space to include the usual formalism, i.e., a set $\Omega$ contains an algebra of events, $F$, for which there exists a probability measure $\mathrm{P}: F \rightarrow[0,1]$ satisfying $\mathrm{P}(\varnothing)=0$ and $\mathrm{P}(\Omega)=1$ and for any disjoint events $\mathrm{A}$ and $\mathrm{B}$ :

$$
P(A \cup B)=P(A)+P(B) .
$$

The triple $(\Omega, F, \mathrm{P})$ is called a probability state space, or 'state space'. See Duffie (1992), Appendix A.
} 
premium investment, i.e., that total amounts paid for all of the contingent claims are equal to the total contingent payouts. And since there is no arbitrage, the pricing system is linear, so that clearly:

$$
\frac{p_{s}}{p_{k}}=\frac{y_{s} p_{s}}{y_{k} p_{k}}=\frac{\left(\mathbf{y}^{T} \mathbf{p}\right) p_{s}}{\left(\mathbf{y}^{T} \mathbf{p}\right) p_{k}} s, k=1,2, \ldots, S
$$

where $p_{s}$ and $y_{s}$ are the sth elements of the vector $\mathbf{p}$ and $\mathbf{y}$, respectively. This condition states that the relative prices of each fundamental state contingent claim is equal to the aggregate relative amounts paid for the respective claims.

In addition, parimutuel principles include a market structure for arriving at the equilibrium prices in which state prices are discovered endogenously via a call auction process. It is the endogenous nature of the price discovery which provides a fundamental connection of parimutuel principles, 'market games' to be discussed next, and the contingent claims and market microstructure research.

The seminal paper of Arrow (1964) demonstrated the equivalence of a competitive exchange economy for contingent commodities with an economy which has a complete and competitive securities market and a spot market in the commodities. In this competitive analysis, the securities market has contingent claims prices which are fixed exogenously. Since prices are fixed, each agent's demand has a negligible effect on the price. Subsequent research has shown that this equivalence result depends crucially on the competitive nature of the securities markets. For example, Peck et al. (1992) show that if the securities market is modelled using a noncooperative market game with endogenous price formation, then the Arrow equivalence result no longer holds. See also Weyers (1999).

The market microstructure literature is largely concerned with endogenous price formation where each agent's demand has a potentially significant impact on the market price. Outside the finance literature, there exists a large body of research utilising the theory of noncooperative market games to model endogenous price formation. An influential paper by Shapley and Shubik (1977) introduced a noncooperative market game for a market with commodities and fiat money but with no uncertainty. In the Shapley-Shubik market game (SSMG), each trader consigns his endowment of each commodity to a trading post dedicated to that commodity. Trade occurs with each trader bidding some of his fiat money to each trading post. When the trading period ceases, the equilibrium price of each commodity is the sum of all the bids in fiat money committed to a trading post divided by the total quantity of commodity consigned to that post. Each trader receives an amount of goods resulting from his bid of fiat money equal to his bid divided by the equilibrium price. Shapley and Shubik (1977) and subsequent papers show that an interior Nash Equilibrium (NE) always exists and that the NE converges to a competitive equilibrium as the economy is replicated. See, for example, Powers et al. (1994).

The SSMG framework has been applied to markets with uncertainty by Peck et al. (1992) and Weyers (1999) as indicated above. Our intent here is to analyse an SSMG market adapted to contingent claims over a state space, i.e., we are interested in the securities market microstructure which may be generally applicable to derivatives and other contingent claims markets. We first show that the SSMG market game with a credit policy restriction on selling is a parimutuel market microstructure. The credit policy, which is defined further below, requires that selling be done on a secured or collateralised basis. 
Proposition 1: A Shapley-Shubik market game for contingent claims within a probability state space with secured selling is a parimutuel market.

Proof: The following notation is required:

$J$ agents indexed $j=1,2, \ldots, J$;

$S$ states indexed $s=1,2, \ldots, S$;

$w^{j}(\mathrm{o})$, initial wealth of agent $j$;

$w_{s}^{j}(f)$, final wealth of agent $j$ in state $s$;

$b_{s}^{j}$, agent $j$ 's bid in dollars for state contingent claim $s$;

$x_{s}^{j}$, agent $j$ 's offer in dollars for insuring contingent claim $s$; and

$p_{s}$, price for state $s$.

First we define the Shapley-Shubik market game model. In the SSMG model, each trader makes bids and offers to each trading post, where each trading post corresponds to a contingent claim within a probability state space. As in the classical SSMG, prices are equal to the ratio of total money bids divided by total commodity consignments or offers for each trading post. For a contingent claims market using the above notation, endogenous price formation therefore takes the following well-known functional form

$$
p_{s} \equiv \frac{\sum_{j=1}^{J} b_{s}^{j}}{\sum_{j=1}^{J} x_{s}^{j}} .
$$

Each state contingent claim price is therefore the sum total of bids in units of money (e.g., dollars) divided by offers in units of money. The offers can be interpreted as sales of the contingent claim, or offers to payout 1 unit of state contingent insurance should the state corresponding to the trading post be realised.

Based upon the preceding notation, the budget constraint for agent $j$ is therefore

$$
w_{s}^{j}(f)=w^{j}(o)-\sum_{s=1}^{S} b_{s}^{j}+\sum_{s=1}^{S} x_{s}^{j} p_{s}+\frac{b_{s}^{j}}{p_{s}}-x_{s}^{j}, \quad \forall s=1,2, \ldots, S .
$$

We assume interest rates are zero and there is no production. Thus, the initial and final wealth in the economy are equal

$$
\sum_{j=1}^{J} w_{s}^{j}(f)=\sum_{j=1}^{J} w^{j}(o) \rightarrow-\sum_{j=1}^{J} \sum_{s=1}^{S} b_{s}^{j}+\sum_{j=1}^{J} \sum_{s=1}^{S} x_{s}^{j} p_{s}+\sum_{j=1}^{J} \frac{b_{s}^{j}}{p_{s}}-\sum_{j=1}^{J} x_{s}^{j}=0, \forall j=1,2, \ldots, J
$$

as implied from the definition of price $p_{s}$,

$$
\sum_{j=1}^{J} \frac{b_{s}^{j}}{p_{s}}-\sum_{j=1}^{J} x_{s}^{j}=0, \quad \forall s=1,2, \ldots, S .
$$

We refer to this condition as the market clearing condition. Summing over $s$ yields the initial (i.e., at the time of premium settlement) market clearing condition that total premiums paid equal total premiums sold, or:

$$
\sum_{j=1}^{J} \sum_{s=1}^{S} b_{s}^{j}-\sum_{j=1}^{J} \sum_{s=1}^{S} x_{s}^{j} p_{s}=0 .
$$


Since all the states comprise a state space, it is required that:

$$
\sum_{s=1}^{S} p_{s}=\sum_{s=1}^{S} \frac{\sum_{j=1}^{J} b_{s}^{j}}{\sum_{j=1}^{J} x_{s}^{j}}=1 .
$$

Clearly, nothing so far developed prevents sellers of claims (i.e., sellers of 'insurance') from defaulting. To address the possibility of default, we assume that the market imposes the following credit restriction on offers of notional insurance.

Define a credit policy as follows: Total offers of notional insurance for any state must be secured by at least the total premiums sold for all of the states, i.e.,

$$
\sum_{j=1}^{J} x_{s}^{j} \leq \sum_{j=1}^{J} \sum_{s=1}^{S} x_{s}^{j} p_{s}, \forall s .
$$

Substituting from the market clearing condition, yields:

$$
\sum_{j=1}^{J} \frac{b_{s}^{j}}{p_{s}} \leq \sum_{j=1}^{J} \sum_{s=1}^{S} b_{s}^{j}, \quad \forall s=1,2, \ldots, S
$$

which yields

$$
\frac{\sum_{j=1}^{J} b_{s}^{j}}{\sum_{j=1}^{J} \sum_{s=1}^{S} b_{s}^{j}} \leq p_{s}, \quad \forall s=1, \ldots, S .
$$

Since the states comprise a probability state space,

$$
\sum_{s=1}^{S} p_{s}=1
$$

Thus, it must be the case that

$$
\frac{\sum_{j=1}^{J} b_{s}^{j}}{\sum_{j=1}^{J} \sum_{s=1}^{S} b_{s}^{j}}=p_{s}, \quad \forall s=1, \ldots, S
$$

which states that the price of each state is equal to the total bids for that state divided by the total bids for all of the states. Thus, the equilibrium pricing condition for the Shapley-Shubik market game for contingent claims requires the relative prices of contingent claims to equal the relative aggregate bids for the respective claims. Since the SSMG is also a call auction market which is self-funding with endogenous price determination, the SSMG for contingent claims is parimutuel.

We can also interpret Proposition 1 in the following way. Each trader who makes an offer for a contingent claim (i.e., a sale of notional insurance) is required to post margin. The margin amount is equal to the premium proceeds. This is a standard practice at most options exchanges and is known as premium margin. Proposition 1 requires that the total 
amount of notional insurance on offer for any state cannot exceed the total premium margin deposited. At most options exchanges, an additional amount of margin related to the risk of the option sold is also required (oftentimes known as additional margin as is the case at Eurex Clearing A.G., the clearinghouse for the Eurex exchange). As no additional margin is required by Proposition 1, we interpret the credit policy to be not overly tight, especially as compared to existing margin mechanisms in use.

Proposition 2: The credit policy constraint requiring the total notional offers of insurance for any state not exceed the total premiums sold can always be satisfied, i.e., it is never binding.

Proof: It can easily be shown that any notional sale can be replicated through a purchase of complementary states within the state space over which claims are traded so that

$$
\sum_{j=1}^{J} x_{s}^{j} \leq \sum_{j=1}^{J} \sum_{s=1}^{S} x_{s}^{j} p_{s}=\sum_{j=1}^{J} \sum_{s=1}^{S} b_{s}^{j}, \forall s .
$$

Consider a notional sale where

$$
x_{s}^{j}>0 \text { and } x_{k}^{j}=0 \text { for } s \neq k .
$$

In this case, agent $j$ sells a claim on state $s$ and on no other state. We use the term replicated sale to denote the strategy of bidding on the complementary states to state $s$ in the following way: ${ }^{5}$

$$
b_{s}^{j}=0 \text { and } x_{k}^{j}=p_{k} x_{k}^{j} \text { for } s \neq k .
$$

The bid on the sth state of the replicated sale is 0 , whereas bids on all other states are non-zero. To ensure the replication is available, we allow the trading post for each state- contingent claim to open with an arbitrarily small bid and offer, i.e.,

$$
\varepsilon_{s}(b) \rightarrow 0, \varepsilon_{s}(x) \rightarrow 0, \forall s=1,2, \ldots, S,
$$

where the arguments $b$ and $x$ indicate the small amounts of existing bids and offers allocated to each state, where these amounts are vanishingly small. ${ }^{6}$ In equilibrium, the profits of a replicated sale are identical to those of the original notional sale,

$$
\begin{aligned}
& w_{s}^{j}(f)=w^{j}(o)-\sum_{s \neq k} p_{s} x_{s}^{j}=w_{s}^{j}(o)-x_{s}^{j}\left(1-p_{s}\right), \\
& w_{s \neq k}^{j}(f)=w^{j}(o)-\sum_{s \neq k} p_{s} x_{s}^{j}+x_{s}^{j}=w^{j}(o)+x_{s}^{j} p_{s}
\end{aligned}
$$

i.e., the final wealth from the replicated sale is identical to the original notional sale for each state. Any notional sale can therefore be replicated into a complementary bid which satisfies the credit policy, and therefore replicated sales are payout-achievable.

We have yet to show that an equilibrium exists with such replication going on during the auction. We turn to this next, and show that any arbitrary number of replications has a fixed-point equilibrium.

\footnotetext{
${ }^{5}$ We note that bidding on all of the states proportional to the price achieves the 'autarky' strategy of effecting no change in each agent's endowments. See Peck et al., (1992).

${ }^{6}$ These small liquidity amounts take the place of the usual SSMG convention that the quantity $0 / 0$ owing to zero bids and offers is equal to 0 . 
Proposition 3: A unique parimutuel equilibrium exists with replicated sales which are used to satisfy the credit policy.

Proof: Consider a notional sale where

$$
x_{s}^{j}>0 \text { and } x_{k}^{j}=0 \text { for } s \neq k .
$$

As indicated above, the replicated sale strategy is

$$
b_{s}^{j}=0 \text { and } x_{k}^{j}=p_{k} x_{k}^{j} \text { for } s \neq k
$$

such that the strategy bid for $s \neq k$ is as follows:

$$
b_{s}^{j}=\frac{\sum_{j}^{J} b_{s}^{j}}{\sum_{j}^{J} \sum_{s}^{S} b_{s}^{j}} x_{s}^{j}=g\left(b_{s}^{j}\right) .
$$

By the Banach Fixed Point Theorem, there exists a fixed point strategy bid for the differentiable function $g$ if there exists a constant $z<1$ such that

$$
\left|g^{\prime}\left(b_{s}^{j}\right)\right| \leq z
$$

Differentiation of $g\left(b_{s}^{j}\right)$ yields:

$$
g^{\prime}\left(b_{s}^{j}\right)=\frac{\sum_{j}^{J} \sum_{s}^{S} b_{s}^{j}-\sum_{j}^{J} b_{s}^{j}}{\left(\sum_{j}^{J} \sum_{S}^{S} b_{s}^{j}\right)^{2}} x_{s}^{j} .
$$

From the market clearing condition, above,

$$
x_{s}^{j} \leq \sum_{j=1}^{J} x_{s}^{j} \leq \sum_{j}^{J} \sum_{s}^{S} b_{s}^{j} .
$$

Together with the obvious

$$
\frac{\sum_{j}^{J} \sum_{s}^{S} b_{s}^{j}-\sum_{j}^{J} b_{s}^{j}}{\sum_{j}^{J} \sum_{s}^{S} b_{s}^{j}}<1
$$

completes the proof. Uniqueness follows from the contraction property of the mapping that leads to the fixed point.

Proposition 4: The SSMG and a parimutuel market have equivalent payouts and first-order optimality conditions.

Proof: Omitted. Available from the authors upon request, as well as in Lange and Economides (2003). ${ }^{7}$

\footnotetext{
${ }^{7}$ Omitted proofs are rather lengthy but are readily available from the authors as well as in Lange and Economides (2001), Appendix.
} 
The first order conditions for the SSMG equilibrium can readily be shown to be:

$$
\frac{q_{s}^{j} u^{\prime}\left(w_{s}^{j}(f)\right)}{q_{k}^{j} u^{\prime}\left(w_{k}^{j}(f)\right)}=\frac{p_{s}}{p_{k}}\left\{\frac{1+\frac{b_{s}^{j}}{B_{s}^{j}}}{1+\frac{b_{k}^{j}}{B_{k}^{j}}}\right\}
$$

which shows that the ratio of expected marginal utilities is equal to the ratio of state contingent prices, i.e., the competitive Arrow-Debreu economy result, multiplied by the term in brackets. These are the same oligopoly conditions derived in ShapleyShubik (1977). The oligopoly conditions depend on the ratio of the size of trader $i$ 's bid in each state to the total amount of bids in that state excluding trader $i$ 's bid. They therefore measure the market's ability to absorb trader $i$ 's bid strategy. As trader $i$ 's bid strategy becomes smaller relative to the total bids on each state, the market approaches the competitively optimal market

\section{Parimutuel Microstructure for Contingent Claims}

In this section we describe the parimutuel microstructure recently used to auction off claims on US economic data releases such as nonfarm payrolls, retail sales, and production indices as well as the Eurozone Harmonised inflation index (ex tobacco prices) and Fannie Mae mortgage pool prepayment speeds. Our goal is to show that the parimutuel market has been designed in a manner which yields contingent claims familiar to market participants in existing derivatives markets. For example, one feature of the parimutuel market microstructure is that all trading strategies are implemented with bids and offers of notional amounts of risky claims. In the Shapley-Shubik contingent claims market game of Section 2, agents implement strategies with offers of notional insurance $x_{s}^{j}$ and bids of premium dollars, $b_{s}^{j}$. Conventionally, however, derivatives contracts are based upon the notional amount to be bought or sold and not denominated in premium dollars. The purchaser of an option, say on the dollar-yen foreign exchange rate, will specify a desired size of the position in notional terms, e.g., 10 million dollars, rather than in terms of the amount of desired premium outlay.

We show next that the parimutuel microstructure allows trading strategies to be implemented with limit orders, whereby a trader may specify a reservation price above (below) which the specified purchase (sale) of a given contingent claim will not be executed. Limit prices have heretofore not been used in parimutuel games.

\subsection{Development of the parimutuel microstructure: definitions and setup}

In this section, we develop the concepts and mathematical notation needed to adequately describe the parimutuel contingent claims microstructure recently used in the capital markets in which trader strategies can be implemented with (1) a notional buy or sell order; (2) a vector of payout ratios corresponding to a range of states (claim bundling); and (3) a limit price.

To begin, we let $U$ denote the value of an underlying variable selected for a parimutuel auction - for example, this variable may be the value of an upcoming release of an economic statistic such as Eurozone Harmonised Inflation index. Before the start of the auction, the strikes for the options to be traded on the underlying are determined. The option strikes are set across the range of likely outcomes of the 
underlying to maximise interest in the claims being offered. Let $k_{1}, k_{2}, \ldots, k_{S-1}$ denote the option strikes and let

$$
k_{1}<k_{2}<\ldots<k_{S-1}
$$

assuming, for simplicity, that the underlying $U$ cannot take on any values between any two strikes. These $S-1$ strikes divide $U$ into $S$ states as shown in the first two columns of Table 1. Associated with each of these $S$ states are $S$ state contingent claims that pay out if and only if that particular state occurs.

Prior to the opening of the parimutuel call auction, the financial intermediary hosting the auction enters orders for each of the $S$ state contingent claims. We refer to these orders as the opening orders. Let the opening order premium be denoted as $\theta_{s}$ for $s=1,2, \ldots, S$ such that

$$
\theta_{s}>0 \quad s=1,2, \ldots, S
$$

Though opening orders can be small relative to the customer orders, opening orders ensure that the parimutuel equilibrium prices are unique.

In the parimutuel market recently run on Eurozone inflation, customers submitted option orders to buy or sell options following standard option market protocols. For notation, assume that customers submit a total of $J$ orders in the auction, indexed by $j=1,2, \ldots, J$. When submitting an order, the customer requests a specific number of contracts, denoted by $r_{j}$. For digital options, we adopt the convention that one contract pays out $\$ 1$ if the digital option expires in-the-money. For vanilla options, we adopt the convention that one contract pays out $\$ 1$ per point that the option is in-the-money. The parimutuel mechanism is novel with respect to existing parimutuel wagering schemes in that customers can specify a limit price for each order, as is done at exchanges including the New York Stock Exchange and the Chicago Board of Trade, i.e., the limit price for a purchase of an option represents the maximum price the customer is willing to pay for the option specified. The limit price for a sell of an option represents the minimum price at which the customer is willing to sell the option. We use $w_{j}$ to denote the limit price for customer order $j$.

The parimutuel mechanism replicates each option using the auction's state contingent claims. For notation, let $a_{j, s}$ represent the notional payout amount of state contingent claim $s$ used to replicate customer order $j$. Recall, for instance, from Table 1 that the first state contingent claim is the digital put struck at $k_{1}$. Therefore, $a_{j, 1}$ is

Table 1

The states, outcomes, and state contingent claims in a PDCA auction.

\begin{tabular}{lll}
\hline State & Outcome & \multicolumn{1}{c}{ State contingent claim } \\
\hline 1 & $U<k_{1}$ & Digital put struck at $k_{1}$ \\
2 & $U=k_{1}$ & Digital range with strikes of $k_{1}$ and $k_{2}$ \\
$\ldots$ & $\ldots$ & $\ldots$ \\
$s-1$ & $U=k_{s-2}$ & Digital range with strikes of $k_{s-2}$ and $k_{s-1}$ \\
$S$ & $U=k_{s-1}$ & Digital range with strikes of $k_{s-1}$ and $k_{s}$ \\
$s+1$ & $U=k_{s}$ & Digital range with strikes of $k_{s}$ and $k_{s+1}$ \\
$\ldots$ & $\ldots$ & $\ldots$ \\
$S-1$ & $U=k_{S-2}$ & Digital range with strikes of $k_{S-1}$ and $k_{S-2}$ \\
$S$ & $U \geq k_{S-1}$ & Digital call struck at $k_{S-1}$ \\
\hline
\end{tabular}


the notional amount of the digital put struck at $k_{1}$ used to replicate order $j$. We require $a_{j, s}$ to be non-negative, and we refer to the vector $\left[a_{j, 1}, a_{j, 2}, \ldots, a_{j, s}\right]$ as order $j$ 's replication weights.

The mechanism determines the replication weights to match order $j$ 's payouts. For example, a digital call pays out a fixed amount if, upon expiration, $U$ is greater than or equal to its strike, denoted as $k_{v}$. If order $j$ is a buy order for this option, then the replicating weights are

$$
a_{j, s}= \begin{cases}0 & s=1,2, \ldots, v \\ 1 & s=v+1, v+2, \ldots, S\end{cases}
$$

This set of state contingent claims pays out if $U$ is greater than or equal to $k_{v}$, matching the payouts of the digital call. If order $j$ is a buy of a vanilla call spread with strikes $k_{v}$ and $k_{w}$ with $k_{v}<k_{w}$, then the replicating weights are ${ }^{8}$

$$
a_{j, s}= \begin{cases}0 & s=1,2, \ldots, v+1 \\ k_{s-1}-k_{v} & s=v+2, v+3, \ldots, w \\ k_{w}-k_{v} & s=w+1, w+2, \ldots, S\end{cases}
$$

For a sell of digital call option struck at $k_{v}$, the customer profits if $U$ is less than $k_{v}$ at expiration. In this case,

$$
a_{j, s}= \begin{cases}1 & s=1,2, \ldots, v \\ 0 & s=v+1, v+2, \ldots, S\end{cases}
$$

The mechanism replicates other options, such as digital puts and vanilla put spreads in a similar fashion.

Let $p_{s}$ denote the equilibrium price of the $s$ th state contingent claim for $s=1,2, \ldots, S$. such that the price of each state contingent claim is positive and that the prices of the state contingent claims sum to unity. Mathematically,

$$
\begin{gathered}
p_{s}>0 \quad s=1,2, \ldots, S \\
\sum_{s=1}^{S} p_{s}=1 .
\end{gathered}
$$

Note that $p_{s}$ has a simple interpretation as the implied probability that state $s$ occurs and the sth state contingent claim expires in-the-money.

Let $\pi_{j}$ denote the equilibrium price for the option requested in order $j$. For simplicity of exposition, we assume here that the auction sponsor does not charge fees. Then

$$
\pi_{j} \equiv \sum_{s=1}^{S} a_{j, s} p_{s}
$$

\footnotetext{
${ }^{8}$ If the underlying $U$ can take on values between adjacent strikes, then the replicating weights for a vanilla call spread become

$$
a_{j, s}= \begin{cases}0 & s=1,2, \ldots, v \\ E\left[U \mid k_{s-1} \leq U<k_{s}\right]-k_{v} & s=v+1, v+2, \ldots, w \\ k_{w}-k_{v} & s=w+1, w+2, \ldots, S\end{cases}
$$

In this case, the replicating weights are based on the conditional expected value of the underlying. 
Each option is priced as the sum of the product of the option's replicating weights and the prices of the state contingent claims. Based on equations (28) and (29), prices can be shown to be arbitrage-free in the sense that it is impossible to combine the options in such a way so as to guarantee a riskless profit. ${ }^{9}$

Let $x_{j}$ denote the equilibrium number of filled contracts for order $j$. If the customer's limit price $w_{j}$ is below the parimutuel equilibrium price $\pi_{j}$, then the order's bid is below the market, and the order receives no fill, so $x_{j}=0$. If the order's limit price $w_{j}$ is exactly equal to the parimutuel equilibrium price $\pi_{j}$, then the order's bid is at the market, and the order may receive a fill, so $0 \leq x_{j} \leq r_{j}$ (recall that $r_{j}$ denotes order $j$ 's requested number of contracts). If the order's limit price $w_{j}$ is above the parimutuel equilibrium price $\pi_{j}$, then the order's bid is above the market, and the order is fully filled, so $x_{j}=r_{j}$. Mathematically, the logic for a buy order is as follows

$$
\begin{aligned}
& w_{j}<\pi_{j} \rightarrow x_{j}=0, \\
& w_{j}=\pi_{j} \rightarrow 0 \leq x_{j} \leq r_{j}, \\
& w_{j}>\pi_{j} \rightarrow x_{j}=r_{j} .
\end{aligned}
$$

The logic for a sell order proceeds in a similar manner. As a final piece of notation, let $M$ denote the total premium paid in the auction, which is the sum of the filled order premiums paid plus the sum of the opening orders.

$$
M \equiv\left(\sum_{j=1}^{J} x_{j} \pi_{j}\right)+\sum_{s=1}^{S} \theta_{s} .
$$

Note that $\mathrm{a}_{j, s} x_{j}$ is the payout order $j$ receives if state $s$ occurs and define $y_{s}$ as

$$
y_{s} \equiv \sum_{j=1}^{J} a_{j, s} x_{j} .
$$

Here, $y_{s}$ is the aggregated customer payouts based on the sth state contingent claim. In matrix form, we can write:

$$
y \equiv A^{T} x
$$

\subsection{Parimutuel Equilibrium Pricing Conditions}

We now proceed to develop the mathematical formulation of the parimutuel equilibrium pricing conditions. After developing the necessary notation, we first prove that existence of a unique parimutuel equilibrium where all orders are market orders, i.e., the limit order constraints are non-binding. We then provide a general parimutuel representation theorem which shows that all parimutuel equilibria in our microstructure are solutions to an eigenvalue problem. In the following section, we then relax the restriction on limit orders and prove the existence of a unique equilibrium for arbitrary limit orders.

\footnotetext{
${ }^{9}$ See, for example, theorem 2 of Ingersoll (1987, p. 55). 
One of the core equilibrium conditions of the parimutuel mechanism is that the system contains sufficient premium to exactly fund any state contingent liability, which can be written as follows

$$
y_{s}+\frac{\theta_{s}}{p_{s}}=M \quad s=1,2, \ldots, S
$$

Here, $y_{s}$ is the total amount of customer payouts filled for state $s$ and the quantity $\theta_{s} /$ $p_{s}$ is the notional payout amount of the opening order for state $s$. Thus, the left hand side of equation (35) represents the total payout that the auction mechanism must make if state $s$ occurs. The right hand side is the total premium collected by the mechanism. Thus, in a parimutuel system, the amount of premium collected is exactly equal to the amount needed to settle the total of filled requests for every state. In this sense, the orders in parimutuel equilibrium are self-hedging.

In another sense, equation (35) relates $y_{s}$, the aggregate order payouts if state $s$ occurs, and $p_{s}$, the price of the sth state contingent claim. For $M$ and $\theta_{s}$ fixed, the greater the payouts $y_{s}$, then the higher $p_{s}$ and the higher the prices of options that pay out if state $s$ occurs. Similarly, the lower the payouts $y_{s}$, then the lower $p_{s}$ and the lower the prices of options that pay out if state $s$ occurs. Thus, in parimutuel pricing equilibrium, the aggregate demand for a particular state is closely related to the price for that state's contingent claim. Finally, let $m_{s}$ denote the total filled premium associated with state contingent claim $s$. Then

$$
m_{s}=p_{s} y_{s}+\theta_{s}, s=1,2, \ldots, S
$$

and equation (35) implies trivially that:

$$
\frac{m_{s}}{m_{k}}=\frac{p_{s}}{p_{k}} \quad s, k=1,2, \ldots, S
$$

which states that the relative premium demand for two states is equal to the relative state prices for those states. We are now in a position to state the following proposition:

Proposition 5: Given demands for orders which are expressed in the form of market orders, there exists a unique parimutuel equilibrium.

Proof: The proof is based upon a simple application of a contracting fixed point theorem by summing up equation (35) over all $S$. The full proof is omitted, but is available from the authors upon request, as well as in Lange and Economides (2003).

We are now in a position to formulate the following theorem regarding the parimutuel market microstructure:

Parimutuel Representation Theorem: All parimutuel equilibria are solutions to the following eigenvalue problem:

$$
\mathbf{H p}=M \mathbf{p} .
$$

Proof: Define the matrix $\mathbf{H}$, which has $S$ rows and $S$ columns where $S$ is, again, the number of defined contingent states in the parimutuel auction, as follows

$$
H \equiv\left[\begin{array}{ccccc}
y_{1}+\theta_{1} & \theta_{1} & \theta_{1} & \cdots & \theta_{1} \\
\theta_{2} & y_{2}+\theta_{2} & \theta_{2} & \cdots & \theta_{2} \\
\vdots & \vdots & \vdots & \cdots & \vdots \\
\theta_{S} & \theta_{S} & \theta_{S} & \cdots & y_{S}+\theta_{S}
\end{array}\right] .
$$


$\mathbf{H}$ is a square matrix with each diagonal entry of $\mathbf{H}$ is equal to $y_{j}+\theta_{s}$. The off-diagonal entries for row $\mathrm{s}$ are equal to $\theta_{s}$ for $\mathrm{s}=1,2, \ldots, \mathrm{S}$. Recall that $\mathbf{p}$ is the vector of length $S$ whose s-th element is $p_{s}$. Note that

$$
\mathbf{H p}=\left[\begin{array}{c}
y_{1} p_{1}+\theta_{1} p_{1}+\theta_{1} p_{2}+\theta_{1} p_{3}+\ldots+\theta_{1} p_{S} \\
\theta_{2} p_{1}+y_{2} p_{2}+\theta_{2} p_{2}+\theta_{2} p_{3}+\ldots+\theta_{2} p_{S} \\
\theta_{3} p_{1}+\theta_{3} p_{2}+y_{3} p_{3}+\theta_{3} p_{3}+\ldots+\theta_{3} p_{S} \\
\ldots \\
\theta_{S} p_{1}+\theta_{S} p_{2}+\theta_{S} p_{3}+\ldots+y_{S} p_{S}+\theta_{S} p_{S}
\end{array}\right]=\left[\begin{array}{r}
y_{1} p_{1}+\theta_{1}\left(\sum_{j=1}^{S} p_{j}\right) \\
y_{2} p_{2}+\theta_{2}\left(\sum_{j=1}^{S} p_{j}\right) \\
y_{3} p_{3}+\theta_{3}\left(\sum_{j=1}^{S} p_{j}\right) \\
\ldots\left(\sum_{m}^{S} p_{m}+\theta_{m}\left(\sum_{j=1}^{S} p_{j}\right)\right.
\end{array}\right] .
$$

Since the $p_{s}$ 's sum to unity (equation 29) we can write

Rewriting equation 35 , we have

$$
\mathbf{H p}=\left[\begin{array}{c}
y_{1} p_{1}+\theta_{1} \\
y_{2} p_{2}+\theta_{2} \\
y_{3} p_{3}+\theta_{3} \\
\cdots \\
y_{m} p_{m}+\theta_{m}
\end{array}\right] .
$$

$$
p_{s} y_{s}+\theta_{s}=M p_{s} \text {. }
$$

The left hand side of this expression is simply the s-th row of Hp. Thus we can write

$$
\mathbf{H p}=M \mathbf{p},
$$

which is the matrix equivalent to equation $41 .^{10}$

The intuition for the eigenvalue representation is that a parimutuel pricing vector must lie in the null space of the net risk, since in a parimutuel mechanism all claim payouts are funded by premiums paid. The net risk of the parimutuel mechanism is:

$$
\text { H }- \text { MI. }
$$

Thus, a pricing vector which lies in the null space of the net risk means that there exists a solution to

$$
(\mathbf{H}-M \mathbf{I}) \mathbf{p}=0,
$$

which is the eigenvalue result. The eigensystem representation makes it easy to see that the parimutuel system has a unique fixed point equilibrium.

\subsection{Parimutuel limit order book equilibrium}

We now introduce limit orders into the parimutuel equilibrium calculations. Limit orders are an important feature of the parimutuel microstructure under discussion.

\footnotetext{
${ }^{10}$ Michael Overton of the Courant Institute of New York University first suggested to us that our parimutuel problem might have an interesting eigensystem representation.
} 
Traditional parimutuel wagering methods do not allow for either notional trading, limit orders, or bundling across risky states. These deficits render the raw parimutuel structure used for wagering less than optimal for use in the capital markets. As previously mentioned, options, futures, and other derivatives contracts are based upon notional contract size, rather than the amount to be invested in such contracts. Furthermore, parimutuel wagering markets expose participants to an excessive amount of transaction risk, as all wagers are executed at prices which vary throughout the auction period and are not known until all wagers have been made. In the capital markets, it is customary to use the device of limit prices to limit transaction risk by which participants can assure themselves that their orders are executed only if the market price is more favourable than their indicated limit price. Finally, parimutuel wagering is normally conducted in an ad hoc manner in which liquidity which could be aggregated within the same state space is fragmented into different 'pools'. For example, wagers on bets for a horse to win are held in a parimutuel pool which is separate from wagers on a horse to 'place'. This means that not only can there be arbitrage opportunities across the separate pools, the pricing within each pool is less efficient due to the disaggregation of liquidity. A viable parimutuel microstructure for the capital markets should aggregate all liquidity within a state space, effectively allowing for the no-arbitrage bundling of any type of contingent claim from the fundamental state claims.

In the previous section, we have shown how a unique parimutuel equilibrium exists where limit prices are not binding, i.e., all orders are market orders. In this section, we prove the existence of a unique parimutuel price equilibrium for limit orders with limit prices that can take any arbitrary value.

3.3.1 Limit order book equilibrium. We regard limit orders as particularly important within the context of the parimutuel mechanism for two reasons. First, they allow mitigation of execution risk owing to changing contingent claim prices during the auction period. In parimutuel wagering, an early bettor subjects himself to the risk that the final odds are lower than when the bet was placed. In our microstructure, we allow traders to control the execution price, effectively substituting a probability of execution at the limit price or better for the continuous change in odds faced by a parimutuel bettor. Second, limit orders are a familiar order execution mechanism in the capital markets which we believe should be incorporated into any viable and practicable microstructure for contingent claims.

With the introduction of limit orders comes the requirement of specifying an objective function for determining, subject to the satisfaction of the limit price constraints, which orders are executed in equilibrium. We choose to maximise the total volume of notional orders that can be executed subject to the limit price constraints. We do this for two reasons. First, we take as our definition of 'liquidity' the maximum amount of notional value that can be accommodated in the auction subject to limit price constraints. Thus, the choice of objective function reflects the definition of liquidity which we are trying to maximise. Second, it is anticipated that the sponsor of the auction will earn transaction fee income as a percentage of notional for each order. Our choice of objective function therefore reflects choosing the set of orders that generate maximum fee income. The optimisation problem can therefore be written in the following form: 
maximize $M$

subject to

$$
\begin{aligned}
& \left.\begin{array}{l}
0<p_{s} \quad s=1,2, \ldots, S \\
\sum_{s=1}^{S} p_{s}=1 \\
\pi_{j} \equiv \sum_{s=1}^{S} a_{j, s} p_{s} \quad j=1,2, \ldots, J \\
w_{j}<\pi_{j} \rightarrow x_{j}=0 \\
w_{j}=\pi_{j} \rightarrow 0 \leq x_{j} \leq r_{j} \\
w_{j}>\pi_{j} \rightarrow x_{j}=r_{j}
\end{array}\right\} j=1,2, \ldots, J \\
& y_{s} \equiv \sum_{j=1}^{J} a_{j, s} x_{j} \\
& \begin{array}{l}
M \equiv\left(\sum_{j=1}^{J} x_{j} \pi_{j}\right) \\
y_{s}
\end{array} \sum_{s=1}^{S} \theta_{s} \\
& y_{s}=M=1,2, \ldots, S
\end{aligned}
$$

Based upon this representation of the parimutuel equilibrium with limit orders, the following proposition can be stated.

Proposition 6: The parimutuel limit order book problem has a unique price equilibrium in state prices when there are non-zero opening orders on each state.

Proof: The proof is based upon fixed point continuation methods and is available from the authors upon request, as well as in Lange and Economides (2003).

In practice, the sponsor of the auction can guarantee that there are nonzero opening orders on each state. Proposition 6 establishes the uniqueness of state prices but does not guarantee the uniqueness of the executed order amounts in equilibrium. The uniqueness of state prices is based upon fixed point methods which are independent of the maximand (in equation 45 , the maximand is $M$, the total premium executed in equilibrium). There are $S-1$ possible degrees of freedom in the executed order amounts in equilibrium, meaning that the maximum number of orders which are partially executed is equal to one less the number of states. As in most microstructure mechanisms, the allocation of partially filled orders is not unique under equilibrium prices but is instead typically determined by 'priority rules', such as time priority or pro rata allocation. In the maximisation of equation 45, the priority rule used for the partially filled orders is to allocate them so as to maximise the total price-weighted volume which is equal to the option premium subject to the unique and already determined equilibrium state prices. Since the maximisation for the partially filled orders is undertaken with respect to fixed equilibrium state prices, the optimisation problem is a linear program. There may be more than one solution for the partially filled orders under this linear program. 
3.3.2 An Example of Limit Order Book Equilibrium. We provide a simple example of the solution of the parimutuel limit order book problem. In our example, we use the following input data:

$$
\begin{aligned}
& S=5 \text { states } \\
& J=8 \text { orders }
\end{aligned}
$$

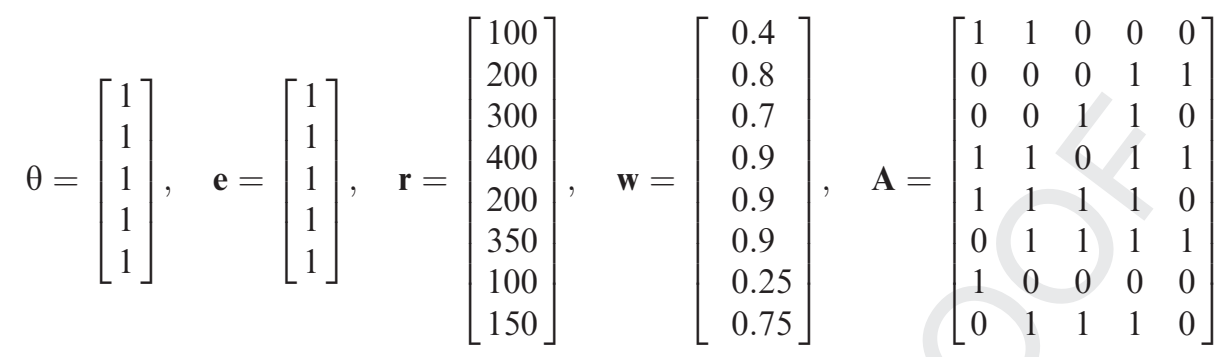

The solution to the optimisation problem is: ${ }^{11}$

$$
\begin{aligned}
\mathbf{x}^{*} & =\left[\begin{array}{r}
100.000 \\
109.560 \\
0.000 \\
0.000 \\
8.571 \\
99.011 \\
100.000 \\
0.000
\end{array}\right], \quad \mathbf{y}^{*}=\left[\begin{array}{l}
208.571 \\
207.582 \\
107.582 \\
217.142 \\
208.571
\end{array}\right], \quad M^{*}=218.571 \\
\mathbf{p}^{*} & =\left[\begin{array}{r}
0.100003859 \\
0.091003287 \\
0.009009933 \\
0.699979066 \\
0.100003856
\end{array}\right] .
\end{aligned}
$$

The interpretation of this example is as follows. There are 5 contingent states representing the fundamental Arrow-Debreu securities. There are 8 submitted orders, as represented in the matrix $\mathbf{A}$, each row of which contains a 1 if the order spans the state represented in the first column, and zero otherwise. For example, the first row of A indicates a digital put option which would pay 1 unit per quantity requested should either of the first two states occur. The quantity requested, or order size, is represented in the vector $\mathbf{r}$. For example, the first row of $\mathbf{r}$ is equal to 100 , indicating that the order size for the digital put spanning the first two states is 100 . The limit prices are contained in the vector $\mathbf{w}$. For example, the first row of $\mathbf{w}$ indicated a limit price of 0.4 for the first order, a digital put spanning the first two states of quantity equal to 100. The limit price indicates that the purchaser of this digital put would like to have his order executed, in equilibrium, at a price of 0.4 per unit of claim (40 in total) or lower. A search procedure is used to find the equilibrium based upon the optimisation in equation (45). The results of solving the equilibrium include the equilibrium

\footnotetext{
${ }^{11}$ Details on the computer algorithm used to solve this example are available from the authors. 
amounts that can be executed for each order (contained in the vector $\mathbf{x}^{*}$ ), the total amount of executed fill for each state (the vector $\mathbf{y}^{*}$ ), the total amount of premium paid for the executed claims in equilibrium based upon their equilibrium prices (the scalar $M^{*}$ ), and the equilibrium prices of the fundamental states (the vector $\mathbf{p}^{*}$ ). The search procedure needs to run to a high level of tolerance which is why $\mathbf{p}$ is reported to a high level of precision.

The equilibrium results can be understood by examining the first three orders. The first order for a digital put spanning the first two states for quantity equal to 100 and a limit price of 0.4 is fully filled, as can be seen from the first row of $\mathbf{x}^{*}$. It is fully filled in equilibrium since the price of a digital put spanning the first two states is the sum of the first two rows of the equilibrium state prices, as shown in $\mathbf{p}^{*}$, which is equal to approximately 0.191 . Since 0.191 is less than the limit price of 0.4 for this order, the order must be fully filled in equilibrium which is the case. The second order spans the last two states, as seen from the second row of $\mathbf{A}$, and therefore should be interpreted as a digital call covering the last two states. From the second rows or $\mathbf{r}$ and $\mathbf{w}$ respectively, the order is one to purchase 200 units at a price of 0.8 or lower. As can be seen from the equilibrium results in the second row of $\mathbf{x}^{*}$, the order is partially filled at 109.56 out of the requested 200. The price of the order is equal to the sum of the last two state prices in $\mathbf{p}^{*}$ or 0.8 . Since the order's equilibrium price is equal to its limit price it may receive a fill anywhere between 0 and the 200 , the requested amount. Finally, order three is a digital range spanning the third and fourth states, for 300 units, at a limit price of 0.7 . As can be seen by adding the third and fourth rows of $\mathbf{p}^{*}$, the equilibrium price of this claim is equal to approximately 0.709 . Since this is higher than the indicated limit price of 0.7 , the order's executed amount in equilibrium is zero, as indicated by the third row of $\mathbf{x}^{*}$.

\section{Parimutuel microstructure: arbitrage and efficiency considerations}

We believe the parimutuel microstructure proposed and analysed in Section 2 compares favourably to other microstructures that may be used for contingent claims trading. We think the parimutuel microstructure under discussion may be superior to dealer-based and currently used exchange structures for a wide variety of risks. We believe that the parimutuel microstructure described in this paper is especially superior for those risks which do not have tradable underlying securities or instruments. We organise our discussion of the benefits of our microstructure into the following six areas: (1) risk-neutrality; (2) the absence of arbitrage; (3) efficiency; (4) price uniqueness; (5) multilateral order matching and; (6) information production.

\subsection{Risk neutrality ${ }^{12}$}

Parimutuel principles entail a self-funded auction of contingent claims: all premium collected, excluding transaction costs, is exactly sufficient to pay for all state con-

\footnotetext{
${ }^{12}$ By 'risk-neutrality' we mean that the parimutuel auction is self-funding in the sense that premium inputs equal state contingent outputs. We do not mean to suggest a connection to the continuous time options literature which is focused on risk-neutral pricing.
} 
tingent payouts. From a dealer perspective, the parimutuel microstructure will be preferable to standard OTC transactions for certain types of derivatives risks. For example, a dealer in fixed income derivatives will likely find the proposed parimutuel microstructure favourable for transacting options on the monthly announcement of the level of the Eurozone Harmonised inflation index since there is no underlying security or hedgeable instrument.

The proposed parimutuel microstructure effects an arbitrage-free and riskless set of contingent claims prices and order executions. Effectively, the mechanism achieves what a dealer would need to do manually through hedging activity in an underlying instrument (where available) and through balancing risk by adjusting prices with trading counterparties to equilibrate net notional transactions across states. We think this simplicity and efficacy of the parimutuel microstructure as adapted to the capital markets is therefore a potentially useful complement to the traditional OTC dealer market structure, especially for types of risks which have no tradable underlying.

We also think that the proposed parimutuel microstructure is superior to conventional exchange-based continuous double auctions for some types of illiquid risks. For example, for a number of years the Chicago Board of Trade (CBOT) has offered options on insurance catastrophe losses as measured by indices published by the Property Claims Service (PCS). The microstructure used to transact these claims is a conventional continuous double auction, i.e., the same mechanism that is used to trade the highly liquid bond futures and options at the CBOT. While there are perhaps reasons why the PCS contracts have failed to attract liquidity which are unrelated to market microstructure, see, e.g., Cummins and Mahul (2000), we believe that the conventional microstructure may be a significant impediment to liquidity, as we discuss further below.

\subsection{Arbitrage-free claims}

A parimutuel system is arbitrage-free in the sense that there exists a positive state price vector which excludes arbitrage. Following the standard definitions (see Ingersoll, 1987, p. 57), we can define the returns table, $Z$, of a parimutuel state space as follows:

$$
\mathbf{Z}=\operatorname{Adiag}(\boldsymbol{\pi})^{-1}
$$

Now, it is well known that if there exists a state pricing vector $\mathbf{p}$ supporting the returns table such that:

$$
\mathbf{Z p}=1
$$

then there exists no arbitrage possibilities in the sense that there exists no investment $\eta$ across the states which solves either: ${ }^{13}$

$$
\begin{aligned}
& \mathbf{e}^{T} \boldsymbol{\eta} \leq 0 \\
& \mathbf{Z}^{T} \boldsymbol{\eta} \geq 0 \text { (one strictly) }
\end{aligned}
$$

\footnotetext{
${ }^{13}$ See Ingersoll (1987), pp. 54-57 for the elementary proof. 
or

$$
\begin{aligned}
& \mathbf{e}^{T} \eta<0 \\
& \mathbf{Z}^{T} \eta \geq 0 .
\end{aligned}
$$

In the proposed parimutuel market microstructure, a definition is that all contingent claim prices are linear combinations of the state prices, i.e.,

$$
\pi=\mathbf{A p} \text {. }
$$

Multiplication of this definition by $\operatorname{diag}(\pi)^{-1}$ establishes that there is a supporting state price vector and that no arbitrage is possible by construction of the parimutuel microstructure.

The claim bundling feature of our parimutuel microstructure by definition rules out arbitrage in the above-defined sense. A market for state contingent claims, even a call auction like the parimutuel mechanism under discussion, need not enforce the no-arbitrage condition explicitly. Namely, we can readily envision a contingent claims market for a state space which can be modelled without such explicit restrictions as follows:

$$
\mathbf{x}^{*}=\underset{\mathbf{x}}{\operatorname{argmax}} \sum_{i=1}^{n} \boldsymbol{x}_{i}
$$

\section{subject to}

$$
\left.\begin{array}{l}
\boldsymbol{w}_{j}<\pi_{j} \rightarrow \boldsymbol{x}_{j}=0 \\
\boldsymbol{w}_{j}=\pi_{j} \rightarrow \mathbf{0} \leq \boldsymbol{x}_{j} \leq r_{j} \\
\boldsymbol{w}_{j}>\pi_{j} \rightarrow \boldsymbol{x}_{j}=r_{j}
\end{array}\right\} \boldsymbol{j}=1,2, \ldots, J
$$

which are limit order conditions without the parimutuel and no-arbitrage price restrictions. In such a market, presumably arbitrageurs would devote capital to ensuring that arbitrage would be excluded from the prices. The parimutuel mechanism enforces the normalisation of state prices and the absence of such arbitrage endogenously within the microstructure.

\subsection{Efficiency of parimutuel price discovery}

The enforcement of the no arbitrage conditions leads naturally to the following welfare result on the efficiency of the parimutuel microstructure compared to a model in which contingent claims are traded separately in a call auction over a state space (the 'trading post' model). Essentially, the parimutuel market as implemented in this paper leads to more efficient (less noisy) prices because the mechanism utilises information on bids and excess demands in all individual markets (trading posts). Put it differently, the parimutuel mechanism as implemented discovers prices that reflect information from all 'trading posts' markets, and this makes the prices reflect more efficiently trading conditions in all posts.

Proposition 7: A parimutuel microstructure discovers prices for contingent claims such that the average order's standard deviation around fair value is less than a microstructure with separate call auction trading posts for each claim. The average order noise savings is equal to

$$
\delta=\alpha \bar{\sigma}\left(\frac{\sqrt{2}-1}{\sqrt{2}}\right) M \cong(0.29) \alpha \bar{\sigma} M
$$


where

$\delta=$ savings due to parimutuel microstructure

$M=$ total premium in system

$\bar{\sigma}=$ average volatility of price error around the true price ('noise volatility')

$\alpha=$ bid/offer spread assumed proportional to average noise volatility

Proof: Omitted. Available from the authors upon request, as well as in Lange and Economides (2003).

We also note that the parimutuel mechanism has an additional efficiency gain over the traditional continuous market because of the time aggregation of orders provided by the call auction itself. ${ }^{14}$

There is suggestive empirical evidence supporting the preceding efficiency result. Gabriel and Marsden (1990, 1991) examine British betting on horses in which parimutuel and bookmakers make prices simultaneously. The bookmakers offer odds on wagers using the 'starting price' odds convention, whereby a bookmaker takes a bet at odds formed by a consensus of bookmakers just before the race is run. Thus, both the parimutuel and starting price odds reflect odds just before the race is run. On the same sample of races, Gabriel and Marsden (1991, Table 1), find that parimutuel returns on the same races are about $28.7 \%$ higher, almost exactly the amount of efficiency owing to the parimutuel system predicted in Proposition 7.

\subsection{Price uniqueness}

The parimutuel microstructure possesses a unique price equilibrium for a given set of opening orders and other orders for contingent claims. Not all microstructures of this class need possess unique equilibrium prices. Consider, in this regard, the following modified microstructure similar to the parimutuel discussed in Section 2 above:

$$
\begin{aligned}
& x^{*}=\underset{x}{\operatorname{argmax}} \sum_{j=1}^{J} x_{j} \\
& \text { subject to } \\
& 0<p_{s}<1 \text { for } s=1,2, \ldots, S \\
& \sum_{s=1}^{S} p_{s}=1 \\
& \left(y^{T} p\right) e=y \\
& \left.\begin{array}{l}
w_{j}<\pi_{j} \rightarrow x_{j}=0 \\
w_{j}=\pi_{j} \rightarrow 0 \leq x_{j} \leq r_{j} \\
w_{j}>\pi_{j} \rightarrow x_{j}=r_{j}
\end{array}\right\} j=1,2, \ldots, J .
\end{aligned}
$$

This microstructure problem is otherwise identical with that of equation 45 except that the parimutuel constraint has been replaced with a weaker constraint in equation 51. The constraint in equation 51 merely requires that the state contingent payouts for each state be equal. This microstructure has some parimutuel features in the sense that

\footnotetext{
${ }^{14}$ See Economides and Schwartz (1995). 
elemental state claims are normalised, exhibit no arbitrage, and relative prices are equal to relative premium investments for each pair of states. Yet, there exists no unique set of state prices which satisfy equation 51. To see this, we consider a state space with three states. Assume that there are 3 orders: a limit buy order for 300 notional covering state 1 at limit price of .3, a limit buy order for 200 notional covering state 2 at limit price of .4, and a limit buy order for 100 notional covering state 3 at limit price of .5 . Clearly, any state probabilities satisfying

$$
\begin{aligned}
& p_{1} \leq .3 \\
& p_{2} \leq .4 \\
& p_{3} \leq .5 \\
& p_{1}+p_{2}+p_{3}=1 \\
& p_{1} y_{1}+p_{2} y_{2}+p_{3} y_{3}=y_{1}=y_{2}=y_{3}
\end{aligned}
$$

is a solution to equation 51, and there are obviously many such solutions, which will satisfy the risk neutrality constraint that all state payouts are equal. For example, one such solution is

$$
\begin{aligned}
& p_{1}=.25 \\
& p_{2}=.25 \\
& p_{3}=.5 \\
& p_{1}+p_{2}+p_{3}=1 \\
& p_{1} y_{1}+p_{2} y_{2}+p_{3} y_{3}=y_{1}=y_{2}=y_{3}=100
\end{aligned}
$$

By contrast, the parimutuel microstructure we propose, embodied as the solution to equation 45 , possesses a unique set of state prices. In the simple example, under consideration, we assume that there exists opening orders on each state of one unit so that

$$
\theta_{1}=\theta_{2}=\theta_{3}=1
$$

The unique solution is:

$$
\begin{aligned}
& p_{1}=.3 \\
& p_{2}=.4 \\
& p_{3}=.3 \\
& p_{1}+p_{2}+p_{3}=1 \\
& y_{1}=y_{3}=100 ; y_{2}=100.8333 \\
& \sum_{s=1}^{3} p_{s} y_{s}+k_{s}=103.333
\end{aligned}
$$

\subsection{Multilateral order-matching}

The parimutuel microstructure we propose is fundamentally a multilateral ordermatching mechanism, by which we mean there exists no requirement of a discrete order match between a single buyer and a single seller. Rather, the order-matching mechanism is inherently 'many-to-one' in the sense that any given contingent claim's 
payout is funded multilaterally by all of the other orders which are filled in equilibrium. We regard this feature as particularly important for claims for which there is no tradable underlying and for which there is not a natural demand for a continuous time market. For example, we regard our market microstructure to be of potential use to trade contingent claims on weather, economic statistic releases, corporate earnings releases, and mortgage prepayment speeds.

The character of our parimutuel microstructure is influenced greatly by the commitment of opening orders, $|\theta|$. For $|\theta|=0$, the microstructure resembles a multilateral matching mechanism in which state prices are normalised, but are not necessarily unique. For $|\theta| \rightarrow \infty$, all orders which have limit prices better than the prices reflected in the opening orders will execute, and will have no impact on the state prices. Thus, large $|\theta|$ will tend to resemble a dealer microstructure in the sense that the dealer may bear significant risk that the distribution reflected in the opening orders distribution will depart from the 'true' distribution. We believe the parimutuel microstructure we propose will tend to be most attractive at small values of $\theta$. We define small such that

$$
1,000<\frac{\sum_{j=1}^{J} \pi_{j} x_{j}}{\sum_{s=1}^{S} k_{s}}<10,000,
$$

i.e., that the ratio of total premium filled in equilibrium to the total amount of opening orders is greater than 1,000 and less than 10,000 .

\subsection{Information production}

Our parimutuel microstructure discovers state prices through a state space partition of an underlying probability distribution. It therefore discovers the probability density function implied by actual trading activity in a transparent and natural way. Some experimental data show that standard parimutuel mechanisms have the ability to aggregate private information (see Plott et al., 1997) into the market density function. We think the implied density produced in our microstructure will be an important and high quality informational externality to the market. The quality of the implied density will be high since the density itself is being traded 'piece by piece' in our microstructure. The density discovered on our microstructure is always enforced to be a probability state space by design. Continuous time options markets, by contrast, produce asynchronous option prices at strikes which have varying liquidity and price noise. As a consequence, the traditional techniques used to extract implied density functions from continuous options data tend to produce very poor information due to data limitations and large noise in continuous time options prices (see Breeden and Litzenberger, 1978).

\section{Conclusion}

A parimutuel market microstructure for contingent claims recently used by Goldman Sachs and Deutsche Bank to offer derivatives on Eurozone Harmonised inflation and other economic indices has been discussed and analysed in this paper. A parimutuel microstructure is a call auction market with special equilibrium pricing conditions on the relative prices of contingent claims. We have shown that the parimutuel contin- 
gent claims mechanism recently employed in the capital markets is quite general, and has its roots in the market games literature.

We have shown how the market microstructure incorporating parimutuel principles for contingent claims which allows for notional transactions, limit orders, and bundling of claims across states is constructed. We have proven the existence of a unique price equilibrium for such a market and suggest an algorithm for computing the equilibrium.

We believe that, for a broad class of contingent claims, the parimutuel microstructure recently deployed offers many advantages over the dominant dealer and exchange continuous-time mechanisms. First, the parimutuel mechanism does not require a discrete order match between two counterparties. Instead, orders are executed multilaterally. All executed orders premium is used to fund all of the contingent in-the-money options, i.e., the payouts. Second, we believe the transparent and straightforward pricing mechanism will be attractive to market participants. We believe that the success of the parimutuel mechanism in the wagering markets can, with the modifications which we have made to the mechanism, be carried over into the capital markets. Third, we believe that the risk neutral and self-hedging nature of the parimutuel mechanism, from the perspective of the broker/dealer or other entity which hosts the auction, offers a superior tradeoff between the risk of derivatives dealing and the compensation for providing liquidity for contingent claims. We believe that the parimutuel microstructure may in fact avoid altogether some of risks inherent in derivatives market-making that periodically result in well-publicised disastrous outcomes. Fourth, we have shown that the parimutuel mechanism as implemented in this paper is more efficient than other trading mechanisms. Finally, we believe that the parimutuel microstructure is ideally suited for completing some markets where there currently is an absence of liquidity, such as contingent claims on mortgage prepayment speeds, corporate earnings, weather, and economic statistics, such as the recent Eurozone inflation auction.

\section{References}

Arrow, K., 'The role of securities in the optimal allocation of risk bearing', Review of Economic Studies, Vol. 31, 1964, pp. 91-96.

Breeden, D., and Litzenberger, R., 'Prices of state contingent claims implicit in option prices', Journal of Business, Vol. 51, 1978, pp. 621-651.

Baron, K., and Lange, J., 'From horses to hedging', Risk Magazine, February 2003.

Black, F., and Scholes, M., 'The pricing of options and corporate liabilities', J. Political Economy, Vol. 81, 1973, pp. 637-654.

Cummins, J. D., and Mahul, O., 'Managing catastrophic risk with insurance contracts subject to default risk', Working Paper (, 2000).

Duffie, D., Dynamic Asset Pricing Theory (Princeton NJ: Princeton University Press, 1992).

Dupont, D. Y., 'Market making, prices, and quantity limits', Working Paper (Board of Governors of the Federal Reserve System, 1995).

Economides, N. and Schwartz, R. A., 'Electronic call market trading', Journal of Portfolio Management, Vol. 21, no. 3, 1995, pp. 10-18.

Gabriel, P. E. and Marsden, J. R., 'An examination of market efficiency in British racetrack betting, J. Political Economy, Vol. 98, 1990, pp. 874-885.

Gabriel, P. E. and Marsden, J. R., 'An examination of market efficiency in British racetrack betting: errata and corrections' J. Political Economy, Vol. 99, 1991, pp. 657-659.

Glosten, L, and Milgrom, P., 'Bid, ask, and transaction prices in a specialist market with heterogeneously informed traders', J. Financial Economics, Vol. 13, 1985, pp. 71-100. 
Groh, C., 'Sequential moves and comparative statics in strategic market games', Working Paper (Department of Economics, University of Mannheim, 1998).

Handa, P. and Schwartz, R. A., 'Limit order trading', Journal of Finance, Vol. 51, 1996, pp. $1835-1861$.

Hausch, D., Lo, V. and Ziemba, W. (eds), Efficiency of Racetrack Betting Markets (San Diego CA: Academic Press, 1994).

Ingersoll, J., Theory of Financial Decision Making (Savage MD: Rowman \& Littlefield, 1987).

Kyle, A. S., 'Continuous auctions and insider trading', Econometrica, Vol. 53, 1985, pp. 1315-1336. 8

Lange, J. and Economides, N., 'A parimutuel market microstructure for contingent claims', Discussion Paper no. EC-01-13 (Stern School of Business, NYU, 2001).

Lange, J. and Economides, N., 'A parimutuel market microstructure for contingent claims', at http://www.stern.nyu.edu/networks/Parimutuel.pdf (2003).

Levin, N., 'Optimal bets in parimutuel systems', Working Paper no. 821/84, The Israel Institute of Business Research, in Hausch et al., 1994.

O'Hara, M., Market Microstructure Theory (Malden MA: Blackwell, 1995).

Peck, J., Shell, K., and Spear, S., 'The market game: existence and structure of equilibrium', J. Math. Econ., Vol. 21, 1992, pp. 271-99.

Plott, C. R., Wit, J. and Yang, W. C., 'Parimutuel betting markets as information aggregation devices', CalTech Social Sciences Working Paper 986 (California Institute of Technology, 1997).

Powers, M., Shubik, M. and Yao, S., 'Insurance market games: scale effects and public policy', Discussion Paper No. 1076 (Cowles Foundation, 1994).

Shapley, L., and Shubik, M., 1977, 'Trade Using One Commodity as a Means of Payment', Journal of Political Economy, Vol. 85:5, 937-968.

Weyers, S., 'Uncertainty and insurance in strategic market games', Economic Theory, Vol. 14, 1999, pp. 181-201. 